\title{
Skin Cancer Detection through Microwaves: Validation on Phantom Measurements
}

\author{
Banafsheh Khalesi \\ Department of Electrical and Electronic Engineering \\ London South Bank University \\ London, UK \\ khalesib@1sbu.ac.uk
}

\author{
Gianluigi Tiberi \\ UBT- Umbria Bioengineering Technologies \\ Spin off of University of Perugia, Perugia, Italy \\ Department of Electrical and Electronic Engineering \\ London South Bank University, London, UK \\ gianluigi@ubt-tech.com
}

\author{
Navid Ghavami \\ UBT- Umbria Bioengineering Technologies \\ Spin off of University of Perugia \\ Perugia, Italy \\ navid.ghavami@kcl.ac.uk
}

\author{
Mohammad Ghavami, Sandra Dudley \\ Department of Electrical and Electronic Engineering \\ London South Bank University \\ London, UK \\ ghavamim@1sbu.ac.uk,dudleyms@1sbu.ac.uk
}

\begin{abstract}
This paper presents the design, fabrication, and demonstration of a heterogeneous phantom simulating human forearm having an inclusion mimicking a skin cancer. Cortical bone, adipose-muscle, and cancerous tumours are simulated at different layers of our proposed phantom. A number of combinations have been tested for tissue-equivalent dielectric properties and the final design has been selected by considering the similarity of their dielectric properties with the respective human tissues. Ultrawideband (UWB) microwave imaging methods are then applied to the phantom. The S21 parameter measurements are collected in an anechoic chamber environment and processed via Huygens principle (HP) technique. The tumour is successfully detected after applying appropriate artefact removal procedure. S21 subtraction using two sets of measurements has been employed to remove artefacts, i.e. the image of the transmitter. The ability to successfully apply HP to detect and locate a skin cancer type inclusion in a multilayerd cylindrical phantom has been proved.
\end{abstract}

Index Terms-Multilayer Phantom, Skin Cancer Imaging, Dielectric Properties, Tissue-equivalent Material.

\section{INTRODUCTION}

The high percentage of annual mortality rate caused by cancer shows the importance of carrying out further research in this area to achieve an appropriate technique for earlystage cancer detection. In general, there are three known types of skin cancer: Squamous Cell Carcinoma (SCC), Basel Cell Carcinoma (BCC), and melanoma. In the majority of cases (especially BCC and SCC), skin cancers are curable if they are diagnosed at an early stage [1]. A wide range of related research works have been performed for detection of a tumour in the early stage. For this purpose, the dielectric properties of human tissues can be used as an effective and accurate indicator for diagnostic purposes [2]. Early studies [3] have indicated that tumour shows a significant dielectric contrast with respect to healthy tissues in the microwave frequency range. On the other hand, in recent years, there has been a growing interest in the development of imaging methods such as those using the UWB microwave imaging technique for medical applications. Its use of non-ionizing signal, low cost, low complexity and its ability to penetrate through mediums are the benefits of UWB technology which has recently attracted the attention of researchers [4]. Microwave imaging makes use of the difference in dielectric properties between normal and malignant skin tissues for detection purposes [4].

Several different techniques have also been proposed for tumour detection, among which is the technique based on Huygens principle (HP) [5], [6]. Up to now, investigations on skin cancer detection has only been studied through analytical simulation [1].

This study describes the phantom construction development for imaging based on microwave technique to detect the skin cancer. Specifically, we propose the design of multilayer anatomical human phantom which consists of cortical bone, adipose $\&$ muscle, and tumour. Several ingredients have been used to mimic different tissues through selection of appropriate materials.

The similarity of the geometric characteristics of each layer of the phantom with the geometry of the desired human forearm has been considered and assessed. In addition, the stability of materials to keep the desired dielectric properties in long-term is investigated. The fabricated phantom has since been used for UWB microwave imaging experiments in an anechoic chamber. It has been shown that Huygens principle technique leads to tumour detection.

\section{Methodology}

\section{A. Phantom Fabrication}

The phantom presented in this paper, which can be used for UWB imaging experiments with working frequency ranging from $1 \mathrm{GHz}$ to $10 \mathrm{GHz}$, has been designed as a multilayer phantom and each layer corresponds to a particular part of the forearm. The human forearm consists of many layers, 
comprising bone, adipose, and muscle. To allow a feasible manufacturing, our proposed phantom has been constructed using three layers, each of which mimics: i) bone, ii) a combination of adipose and muscle tissue, iii) a cancerous tumour represented by an inclusion inside the adipose and muscle layer. The dielectric constant of each layer is given in Table I, where the values have been derived from [7]. A specific recipe has been considered for each layer with the aim of simulating dielectric parameters of human tissues.

TABLE I

DIELECTRIC CONSTANT AND CONDUCTIVITY AT THE FREQUENCY OF 5.5 $\mathrm{GHz}[7]$

\begin{tabular}{|c|c|c|}
\hline Microwave frequency (5.5 GHz) & Permittivity & Conductivity (S/m) \\
\hline Bone (Cortical) & 10 & 0.96 \\
\hline Adipose \& Muscle & 27 & 2.14 \\
\hline Tumour & 50 & 3 \\
\hline
\end{tabular}

Our proposed complex phantom contains the following parts: layer of bone, a combination of adipose \& muscle $(50 \%$ $50 \%$ ) and tumour.

As Fig. 1 and Table II show, three different volumes of the cylindrically shaped container have been used for constructing the phantom and then they have been filled up with different specific materials to represent bone tissue, a combination of adipose \& muscle, and tumour. In addition, the external plastic cylinder layer has been assumed to be the skin layer.

TABLE II

PHANTOM LAYERS' DESIGN HEIGHT AND SIZE

\begin{tabular}{|c|c|c|}
\hline Tissue layers of the phantom & Radius (cm) & Height (cm) \\
\hline Adipose \& Muscle & 5.5 & 13 \\
\hline Bone & 3.5 & 9 \\
\hline Tumour & 0.75 & 7 \\
\hline
\end{tabular}

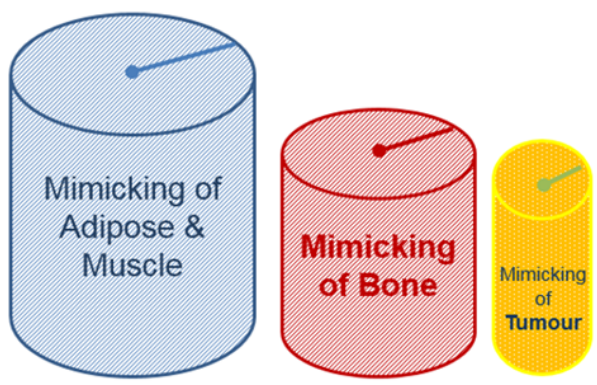

Fig. 1. Design of different layers of phantom.

Constructing the phantom has been performed through the following steps. The small cylindrically shaped container has been filled up with tumour equivalent material. The medium cylindrically shaped container has been filled up with bone equivalent material. Then the tumour and the bone have been placed in the large cylindrically shaped plastic container. In this step, adipose and muscle equivalent materials have been poured to completely fill the remaining space surrounding the bone and the tumour, as shown in Fig. 2.

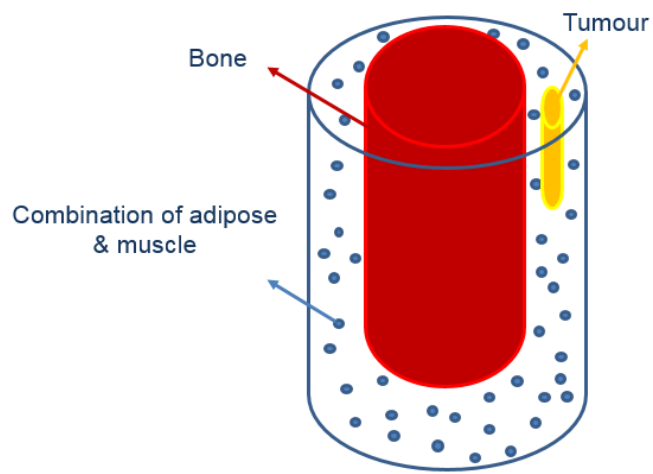

Fig. 2. Design of the proposed phantom.

Each layer of the phantom has been constructed by mixing constituent materials with the appropriate ratio. Bone cortical has been designed to be solid, while the combination of adipose \& muscle have been designed as semi-solid and the tumour has been designed a liquid. Moreover, the recipes have been selected by considering the similarity of its dielectric properties and geometric characteristics with human tissue.

Various recipes for tissue-equivalent dielectric properties have been tested to find the best one. Then the best recipe for each layer has been tried more than twice before being finalized. The permittivity properties have been measured more than three times and the average value has been taken. The dielectric constant of each recipe has been checked by using the device described in the next sections. The recipes' alteration concluded when the obtained permittivity value found via the measurement device was the same as the desired permittivity value given in Table I. Table III shows the required ingredients for each recipe which is the most appropriate one for mimicking each tissue due to the fact that its recipe gives the correct dielectric constant value [8], [9].

TABLE III

TISSUE MIMICKING RECIPES; ALL THE INGREDIENTS ARE GIVEN IN GRAMS.

\begin{tabular}{|c|c|c|}
\hline Ingredient & Bone & Adipose \& Muscle \\
\hline Deionized water & 10.5 & - \\
\hline Flour & 50 & 30 \\
\hline Vegetable Oil & 30 & 25 \\
\hline Sugar & 7.2 & - \\
\hline Salt & 0.2 & - \\
\hline Detergent & - & 12 \\
\hline
\end{tabular}

The detailed fabrication method for our proposed complex phantom is as follows.

Bone cortical:

1) In the bowl, mix the vegetable oil and flour for 10-15 minutes until all the oil was absorbed by the flour. 
2) Then, mix the sugar with deionized water until the sugar was dissolved in the water.

3) Add the solution into the bowl and mix them until it formed a uniform shape.

Adipose \& Muscle:

1) Flour and vegetable oil were mixed.

2) Then, detergent was added to the mixture and we continued to stir the mixture until it formed a uniform shape.

3) The mixture was put in the fridge overnight.

Finally, the tumour has been mimicked through a mixture of Glycerol and water with ratios of $60 \%$ and $40 \%$, respectively as given in [10].

\section{B. Permittivity Measurement Device}

The dielectric properties of the tissue-representing phantom materials were measured using the Epsilon dielectric measurement device (Biox Epsilon Model E100 manufactured by Biox System Ltd Company). The Epsilon is an instrument for contact system using a fingerprint sensor that responds to capacitance. It can be used for measurements on isolated soft solids and liquids.

It consists of a base for mounting the probe with its sensor surface horizontal and facing upwards as shown in Fig. 3. The instrument is calibrated using materials of known dielectric permittivity (dielectric constant $\varepsilon$ ), to ensure consistent measurements from instrument to instrument and from time to time. The device does not give any information about conductivity.
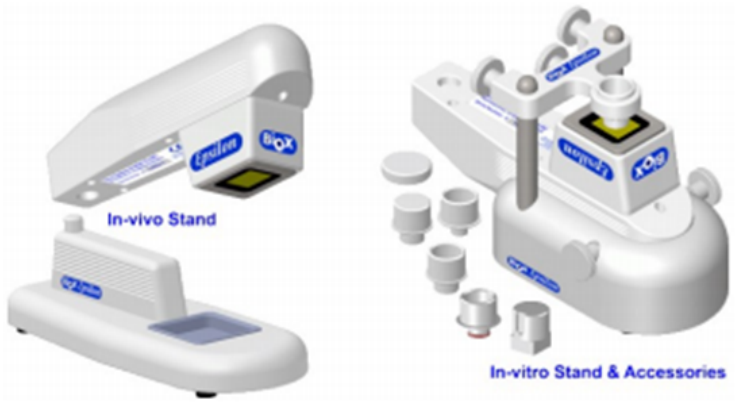

Fig. 3. Epsilon measurement device.

\section{Measurements in an Anechoic Chamber}

Frequency-domain UWB measurements were performed in an anechoic chamber using a Vector Network Analyzer (VNA) arrangement to obtain the transfer function (S21) between two antennas. PULSON antennas, vertically polarized and omni-directional in the azimuth plane were used in freespace, after calibration. The phantom was placed in the center of a rotatable table. Figs. 4 and 5 show the fabricated phantom placed inside the anechoic chamber. In order to exploit the variation of signals at different frequencies, the complex S21 values were recorded over a wide frequency range of 1-10 $\mathrm{GHz}$, employing a frequency step of $5.6 \mathrm{MHz}$. For each set of measurements, transmitting antenna has been fixed at approximately $10 \mathrm{~cm}$ away from the center of table whilst the position of the receiver antenna has been considered $6.5 \mathrm{~cm}$ away from the center of table and mounted on a computercontrolled rotating stage with 6 degrees of angular resolution (number of points NPT $=60$ ).

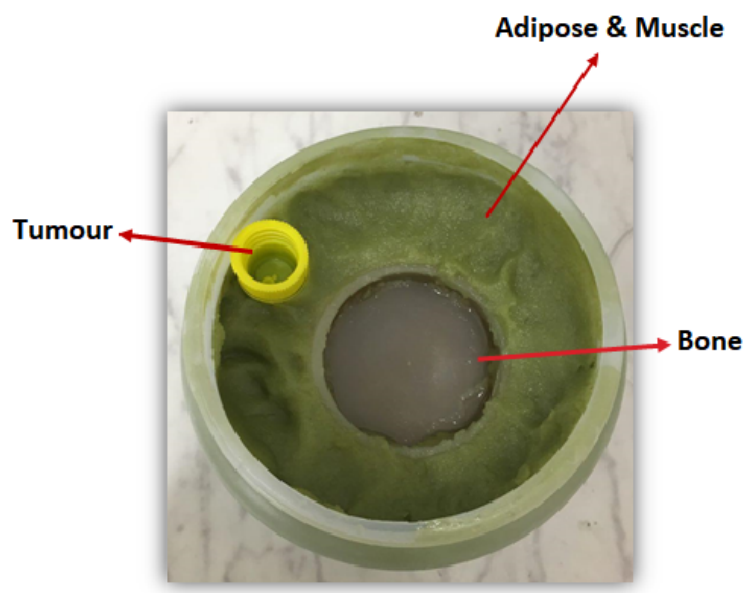

Fig. 4. Fabricated phantom.

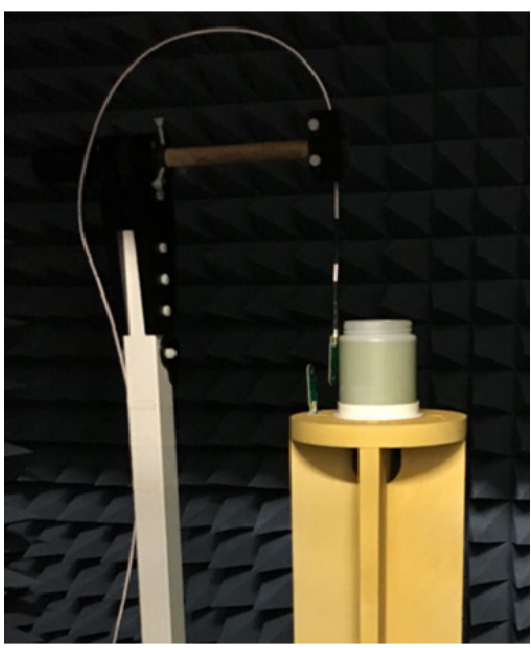

Fig. 5. Position of the phantom inside the anechoic chamber.

The procedure has been repeated for 6 transmitting positions, divided into 2 triplets displaced at $0^{\circ}$, and $90^{\circ}$; each triplet contains 3 transmitting position displaced $5^{\circ}$ from each other such that the first triplet includes positions $0^{\circ}$, $5^{\circ}$, and $10^{\circ}$ while the second triplet includes positions $90^{\circ}$, $95^{\circ}$, and $100^{\circ}$. Note that, rather than moving the transmitting antenna, the 6 transmitting positions have been synthetized by appropriately rotating the phantom.

Fig. 6 shows the measurement set up diagram and the position of phantom with respect to the transmitting position of $0^{\circ}$. 


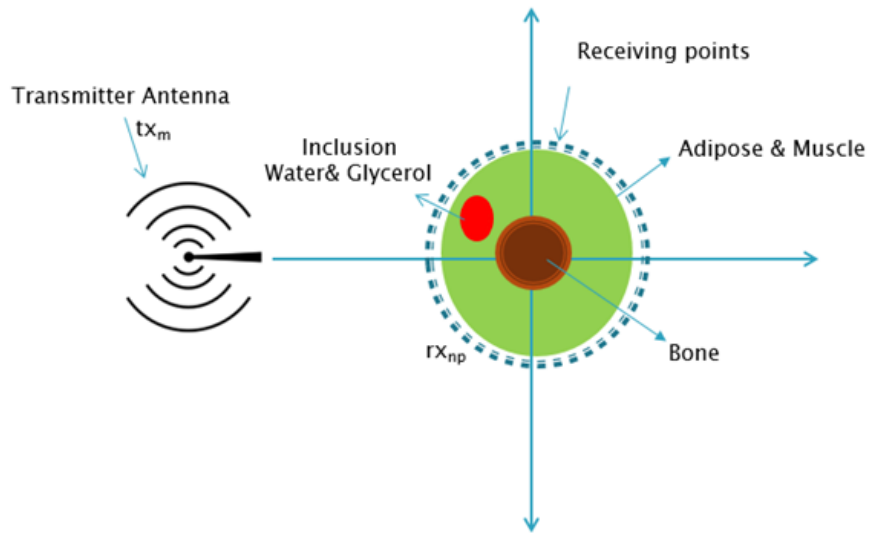

Fig. 6. Pictorial view of the measurement setup.

\section{Imaging Procedure}

The measured signal, i.e., The complex S21 of the VNA are processed through HP procedure. It has been shown in [5], [11] and [12] that HP is able to identify the presence and location of the dielectric discontinuities inside an object by using only the outside field measurement.

In more detail, referring to Fig. 6, suppose we measure the S21 at the points $\mathrm{rx}_{n p} \equiv\left(a_{0}, \phi_{n p}\right) \equiv \vec{\rho}_{n p}$, displaced along a circular surface having radius $a_{0}$ :

$$
\left.S 21_{\mathrm{t}_{m}}^{\mathrm{known}}\right|_{\mathrm{r}_{n} x_{n}}=S 21_{n p, \mathrm{t}_{m}}^{\mathrm{known}} \quad \text { with } \quad n p=1, \ldots, N_{\mathrm{PT}}
$$

We calculate the field inside the cylinder as superposition of the fields radiated by the $N_{\mathrm{PT}}$ observation points of eq. (1):

$$
E_{\mathrm{HP}, 2 \mathrm{D}}^{\mathrm{rcsst}}\left(\rho, \phi ; \mathrm{tx}_{m} ; f\right)=\Delta_{s} \sum_{n p=1}^{N_{P T}} S 21_{n p, \mathrm{tx}_{m}}^{\mathrm{known}} G\left(k_{1}\left|\vec{\rho}_{n p}-\vec{\rho}\right|\right)
$$

where $(\rho, \phi) \equiv \vec{\rho}$ is the observation point, $k_{1}$ represents the wave number in free space (due to the presence of receiving antenna in free space) and $\Delta_{s}$ is the spatial sampling. In eq. (2), the string "rcstr" is used to indicate the "reconstructed" internal field, while the string HP indicates that a HP based procedure will be employed. In eq. (2), the Greens function $G$ is used to propagate the field. Assuming we use $N_{F}$ frequencies, it follows that the intensity of the final image $I$ can be obtained through the following equation, i.e. by summing incoherently all the solutions:

$$
I(\rho, \phi)=\sum_{i=1}^{N_{F}}\left|E_{\mathrm{HP}}^{\mathrm{rcstr}}\left(\rho, \phi ; \mathrm{tx}_{m} ; f_{i}\right)\right|^{2}
$$

To remove artefacts, i.e., the images of the transmitter [5], [11], [12], we employ here the subtraction between S21 obtained using two sets of measurements of the same triplet, using the formula:
$E_{\mathrm{HP}, 2 \mathrm{D}}^{\mathrm{rcstr}}\left(\rho, \phi ; \mathrm{tx}_{m} ; f\right)=\Delta_{s} \sum_{n p=1}^{N_{P T}}\left(S 21_{n p, t \mathrm{x}_{m}}^{\mathrm{known}}-S 21_{n p, \mathrm{tx}_{m^{\prime}}}^{\mathrm{known}}\right) G\left(k_{1}\left|\vec{\rho}_{n p}-\vec{\rho}\right|\right)$

with $\mathrm{tx}_{m}$ and $\mathrm{tx}_{m^{\prime}}$ belonging to the same triplet.

\section{Results AND Discussions}

The permittivity of the proposed recipe for each layer of the phantom has been measured separately by placing a small sample of the prepared mixture on the surface of the Epsilon device. Fig. 7 shows the method of measuring the samples' permittivity.

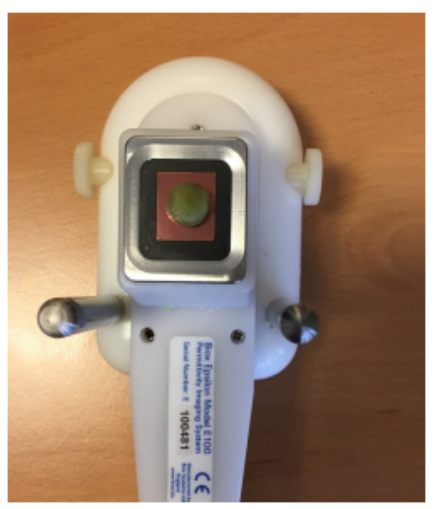

Fig. 7. Measuring permittivity of the mixture.

The recipe for each layer was finalized when the obtained permittivity was very close to the real permittivity of human tissue (previously shown in Table I). The obtained permittivity value of the final recipe for each layer of phantom has been collected in Table IV.

TABLE IV

OBTAINED PERMITTIVITY FOR EACH LAYER OF PHANTOM.

\begin{tabular}{|c|c|}
\hline Layer of pahntom & Our receipe's permittivity value \\
\hline Bone & 10.85 \\
\hline Adipose \& Muscle & 25.26 \\
\hline Tumour & 51.70 \\
\hline
\end{tabular}

As can be observed from the results, an excellent agreement was found between the value given in Table IV and the corresponding values given in Table I. It is important to highlight that the measurement device does not give the dielectric value at the frequency of the interest but gives the permittivity values at frequency $=0 \mathrm{~Hz}$. However, it is still suitable for recipes which consist of ingredients that do not experience any change with frequency variation such as oil and flour. Accordingly, since the muscle and bone recipes are constructed mainly by a high percentage of oil and flour, therefore, the obtained measured permittivity with this device at zero frequency has been considered equivalent with its permittivity at a frequency of $5.5 \mathrm{GHz}$. This does not hold true for glycerol and water mixture [10], however, the permittivity of such mixture can be derived from [10], where the conductivity is also given. 
Figs. $8 \mathrm{a}, 8 \mathrm{~b}, 8 \mathrm{c}$, show the microwave images phantom when employing eq (2) for individual transmitting positions of $0^{\circ}$, $5^{\circ}$, and $10^{\circ}$, respectively. All the images show the transmitter artefact, which masks the inclusion.

Figs. 8d, 8e, 8f, depict the microwave images of the phantom when employing eq (4), i.e. using the difference between measurements obtained with transmitting position $0^{\circ}$ and $5^{\circ}$, $5^{\circ}$ and $10^{\circ}, 0^{\circ}$ and $10^{\circ}$, respectively. The inclusion is now clearly detectable in the correct position; in addition, since it is detectable in Figs. 8d, 8e, 8f in almost the same position, we can conclude that it is not an artefact.

Fig. 9 refers to the second triplet, and confirms the previous results. Specifically, the inclusion shown in Figs. 9d, 9e, 9f, appears here with a $90^{\circ}$ rotation with respect to Figs. 8d, 8e, and 8f. This is in agreement with our experiments, since the transmitting positions have been synthesized by appropriately rotating the phantom.

\section{CONCLUSIONS}

In this paper, the development of a realistic human forearm phantom for skin cancer detection has been described. We have fabricated a phantom using dedicated recipes mimicking layers of cortical bone, adipose \& muscle and cancerous tumour. The combination of adipose and muscle has been considered here using a ratio of $50 \%-50 \%$; future research will be focused on the development of other phantoms that would realize different combination of adipose and muscle, analyzing the impact of the combination ratio in tumour detection. Various recipes were tested for tissue-equivalent dielectric properties and the final recipes have been selected by considering the similarity of its dielectric properties with the human tissues. The phantom is then used for UWB microwave imaging using the Huygens principle. Detection of the tumour has been achieved after removing the artefacts, i.e. the images of the transmitter, through employing a subtraction between S21 obtained using two sets of measurement. This paper verifies that HP can successfully be used to detect and locate a skin cancer type inclusion in a multilayer cylindrical phantom.

\section{ACKNOWLEDGMENT}

The authors would like to thank Dr. Perry Xiao for providing the permitivitty measurement device used in this research.

\section{REFERENCES}

[1] N. Ghavami, G. Tiberi, M. Ghavami, S. Dudley and M. Lane, "Huygens principle based UWB microwave imaging method for skin cancer detection”, 2016 10th International Symposium on Communication Systems, Networks and Digital Signal Processing (CSNDSP), Prague, 2016, pp. $1-4$.

[2] S. I. Alekseev and M. C. Ziskin, "Human skin permittivity determined by millimeterwave reflection measurements", Bioelectromagnetics, 28, pp. 331-339, July 2007.

[3] W. T. Joines, R. L. Jirtle, M. D. Rafal, and D. J. Schaefer, "Microwave power absorption differences between normal and malignant tissue", Int. J. Rad. Oncol. Biol. Phys., vol. 6, pp. 681687, 1980.

[4] E. M. Staderini, "UWB radars in medicine", in IEEE Aerospace and Electronic Systems Magazine, vol. 17, no. 1, pp. 13-18, Jan. 2002.

[5] G. Tiberi, N. Ghavami, D. J. Edwards and A. Monorchio, "Ultrawideband microwave imaging of cylindrical objects with inclusions", in IET Microwaves, Antennas \& Propagation, vol. 5, no. 12, pp. 1440-1446, September 162011.
[6] N. Ghavami, G. Tiberi and D. J. Edwards, "Huygens Principle-based approach for UWB medical imaging", 2011 8th European Radar Conference, Manchester, 2011, pp. 369-372.

[7] C. Gabriel, S. Gabriel, and E. Corthout, "The dielectric properties of biological tissues: I. literature survey", Physics in Medicine and Biology, vol. 41, no. 11, pp. 22312249,1996 .

[8] A. Sani, M. Rajab, R. Foster and Y. Hao, "Antennas and Propagation of Implanted RFIDs for Pervasive Healthcare Applications", in Proceedings of the IEEE, vol. 98, no. 9, pp. 1648-1655, Sept. 2010.

[9] S. Symeonidis, W. G. Whittow and C. Panagamuwa, "Design and characterization of a three material anatomical bone phantom for implanted antenna applications", Loughborough Antennas \& Propagation Conference (LAPC 2017), Loughborough, 2017, pp. 1-5.

[10] P. M. Meaney, C. J. Fox, S. D. Geimer and K. D. Paulsen, "Electrical Characterization of Glycerin: Water Mixtures: Implications for Use as a Coupling Medium in Microwave Tomography", in IEEE Transactions on Microwave Theory and Techniques, vol. 65, no. 5, pp. 1471-1478, May 2017.

[11] N. Ghavami, G. Tiberi, D. J. Edwards and A. Monorchio, "UWB Microwave Imaging of Objects With Canonical Shape", in IEEE Transactions on Antennas and Propagation, vol. 60, no. 1, pp. 231-239, Jan. 2012.

[12] G. Tiberi, N. Ghavami, D. J. Edwards and A. Monorchio, "A mode matching - Bessel functions based approach for UWB microwave imaging", 2010 IEEE Antennas and Propagation Society International Symposium, Toronto, ON, 2010, pp. 1-4. 


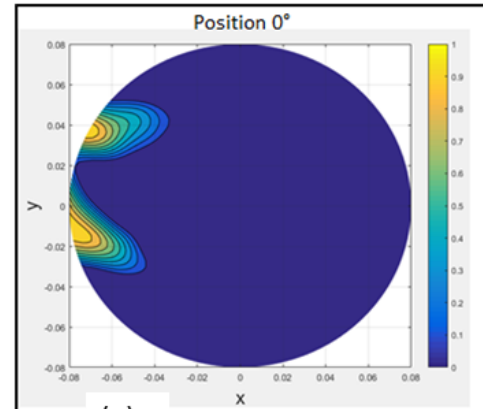

(a)

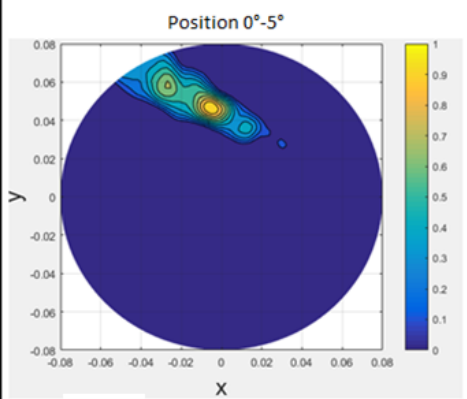

(d)

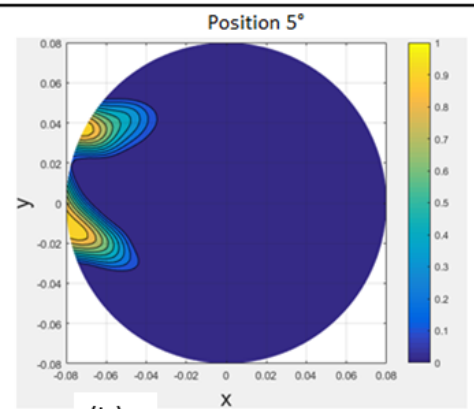

(b)

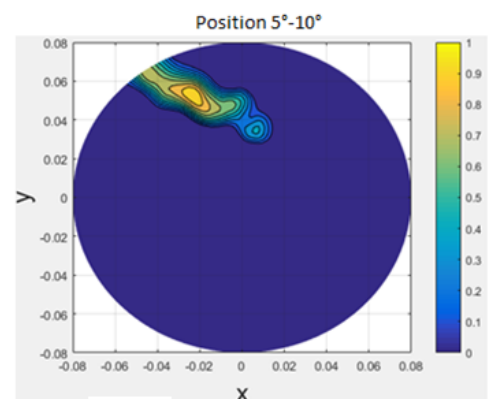

(e)

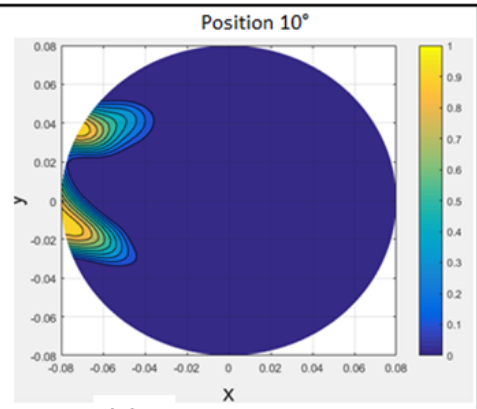

(c)

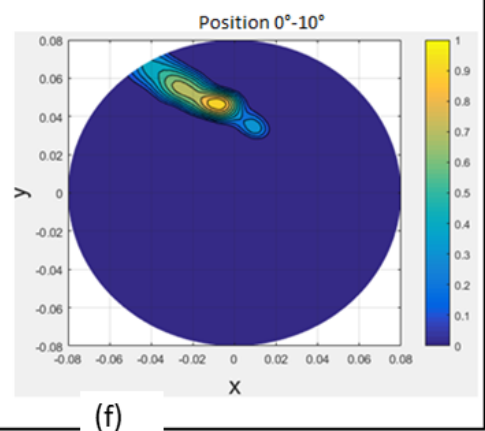

(f)

Fig. 8. Microwave images obtained of the fabricated phantom on the first triplet measurement.

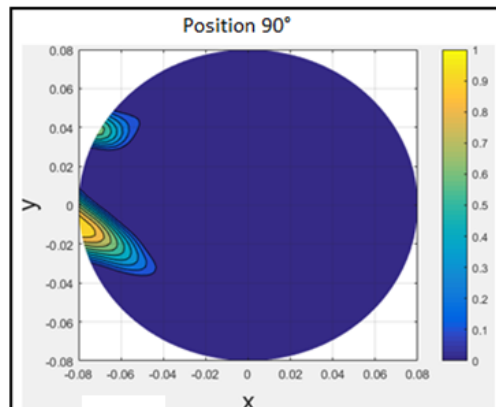

(a)

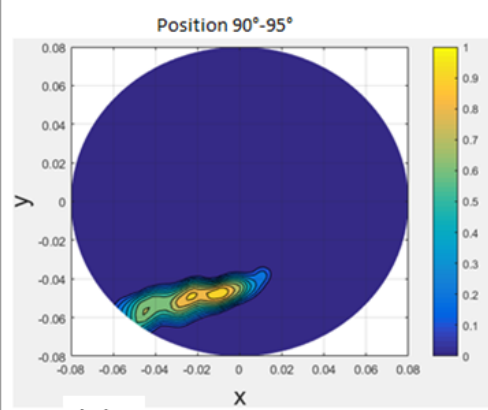

(d)

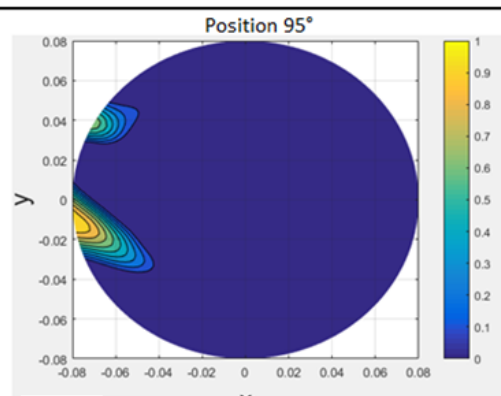

(b)

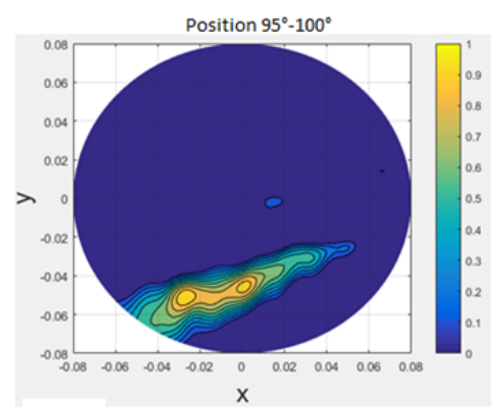

(e)

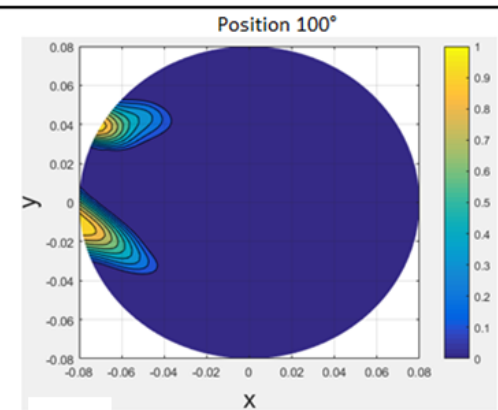

(c)

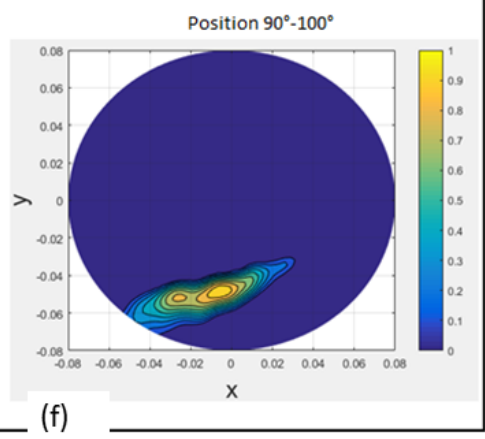

Fig. 9. Microwave images obtained of the fabricated phantom on the second triplet measurement. 